\title{
Could arterial stiffness be early reversible target organ damage test in childhood hypertension?
}

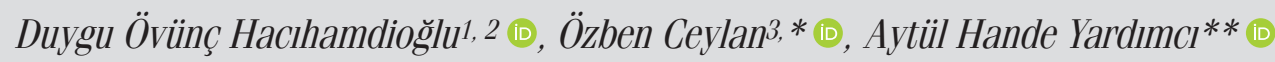

${ }^{1}$ Division of Pediatric Nephrology, Department of Pediatrics, Faculty of Medicine, Bahçeşehir University, Medical Park Göztepe Hospital; İstanbul-Turkey

Divisions of 2Pediatric Nephrology, and ${ }^{3}$ Pediatric Cardiology, Department of Pediatrics, University of Health Sciences,

Süleymaniye Women Maternity and Child Diseases Training and Research Hospital; İstanbul-Turkey

Departments of *Pediatric Cardiology, and **Radiology, University of Health Sciences, İstanbul Training and Research Hospital; İstanbul-Turkey

\section{ABSTRACT}

Objective: The recommended treatment for hypertension (HTN) in children has been revised recently. This study aimed to present the changes in target organ damage (TOD) and arterial stiffness parameters after treatment in children with primary HTN who were managed in accordance with the 2016 European Society of Hypertension Guidelines.

Methods: Patients with primary HTN included in this study were newly diagnosed, untreated, and were followed-up for a minimum of 6 months. HTN was confirmed by 24-h ambulatory blood pressure monitoring (ABPM). All patients underwent the following assessments: anthropometrical measurements of body mass index (BMI), carotid intima-media thickness (cIMT), left ventricular mass index (LVMI), plasma creatinine, urea, electrolytes, uric acid, fasting plasma glucose, blood lipids, urinalysis, urine culture, and first morning urine albumin tocreatinine ratio. The ABPM device performed measurements such as central blood pressure (cBP) and pulse wave velocity (PWV).

Results: Thirty-two of 104 patients were enrolled. Seventeen patients were male, and $53 \%$ were obese. Compared with pretreatment, creatinine, urea, systolic BP (SBP), diastolic BP (DBP), systolic load, diastolic load, central SBP (cSBP), cSBP z score, cDBP, and PWV z score decreased, whereas LVMI and BMI z scores were unchanged.

Conclusion: After BP improvement, while LVMI did not regress, the cSBP, cSBP z, and PWV z score values, which are markers of arterial stiffness, regressed. This supports the corrective effect of BP control on the cardiovascular system even in a short-term follow-up. Further longitudinal studies are needed for the assessment of BP control on arterial stiffness in childhood.

Keywords: hypertension, children, left ventricular hypertrophy, arterial stiffness

Cite this article as: Hacıhamdioğlu DÖ, Ceylan Ö, Yardımcı AH. Could arterial stiffness be early reversible target organ damage test in childhood hypertension? Anatol J Cardiol 2021; 25: 496-504.

\section{Introduction}

The recommended treatment for hypertension (HTN) in children has been revised recently $(1,2)$. In particular, The European Society of Hypertension Guidelines 2016 (ESHG16) recommends further investigation into central blood pressure (cBP) as a parameter for stiffness of the arteries. Left ventricular ejection generates a pulse wave that travels forward through the arterial tree. Pulse wave velocity (PWV) is based on arterial stiffness. Increased stiffness causes the reflected wave to arrive earlier in the central aorta and augment cBP in the systole (3). We followed-up patients who attended the outpatient clinic with an arterial stiffness test according to the
ESHG16. The primary aim of the study was to present the changes in TOD and arterial stiffness parameters after treatment in children with primary HTN.

\section{Methods}

\section{Patients}

This was a retrospective observational study. It examined children with HTN attending the pediatric nephrology outpatient clinic of our hospital. The study was approved by the Local Ethics Committee (2011-KAEK-50) and was conducted in accordance with the Declaration of Helsinki. Informed consent was obtained from all participants. 


\section{HIGHLIGHTS}

- Antihypertensive treatment can reduce blood pressure (BP) in children.

- However, left ventricular mass index may not regress after 6 months of follow-up.

- In contrast, central systolic BP and pulse wave velocity z scores may regress in parallel with reduction in $\mathrm{BP}$.

- These suggest that vascular function tests may detect an earlier response of BP regression in cardiovascular system in children.

HTN was confirmed by 24-h ambulatory blood pressure monitoring (ABPM). HTN was defined as a mean daytime or nighttime blood pressure (BP) $\geq$ the $95^{\text {th }}$ percentile for pediatric ambulatory normal values (4). Based on the ESHG16, the 95th percentile has been used as a threshold for HTN in children and adolescents provided that the values are less than the acceptance criteria for adults (24-h ABPM, $130 / 80 \mathrm{~mm} \mathrm{Hg}$; daytime BP, $135 / 85 \mathrm{~mm} \mathrm{Hg}$; nighttime BP, $125 / 75 \mathrm{~mm} \mathrm{Hg}$ ). HTN was classified as stage 1 HTN (95 ${ }^{\text {th }}-99^{\text {th }}$ percentile plus $5 \mathrm{~mm} \mathrm{Hg}$ ) and Stage 2 HTN (> the 9gth percentile plus $5 \mathrm{~mm} \mathrm{Hg}$ ). HTN in patients aged 16 years or older was graded as that for adults. For patients aged $1-15$, normotension was defined as systolic and/or diastolic BP $<$ the $90^{\text {th }}$ percentile with respect to age, sex, and height. For patients aged 16 or older, normotension was defined as $<130 / 85$ $\mathrm{mm} \mathrm{Hg}$ and confirmed by ABPM. While obesity was defined as body mass index $(\mathrm{BMI}) \geq$ the $95^{\text {th }}$ percentile for age and sex, overweight was defined as having BMI $\geq$ the $85^{\text {th }}$ - and $<$ the $95^{\text {th }}$ percentile for age and sex. Non-obesity was defined as BMI < the $85^{\text {th }}$ percentile for age and sex. All patients underwent the following assessments: anthropometrical measurements of $\mathrm{BMI}$, carotid intima-media thickness (cIMT), left ventricular mass index (LVMI), plasma creatinine, urea, electrolytes, uric acid, fasting plasma glucose, blood lipids (total cholesterol and triglycerides), urinalysis, urine culture, and first morning urine albumin to creatinine ratio. The inclusion criteria were as follows: newly diagnosed patients with untreated primary HTN with a follow-up period of at least 6 months. Exclusion criteria were as follows: the presence of any significant chronic disease such as diabetes, chronic kidney disease, congenital kidney and urinary tract abnormality, and any acute diseases (including infections) in the 6 weeks preceding enrollment; use of any medications during the study or in the preceding 6 weeks, requiring chronic pharmacological treatment; and incomplete data.

After diagnosis, we advised all patients about lifestyle changes according to guidelines and recommendations (1). Antihypertensive treatment was initiated in those who presented with symptomatic or TOD. Antihypertensive treatment was also initiated in those whose blood pressure was not controlled by lifestyle changes after 2 months and who thus remained symptomatic. Our patients were prescribed the renin-angioten- sin-aldosterone system (RAAS) blocking drugs (enalapril, at a dose of $0.2-0.6 \mathrm{mg} / \mathrm{kg}$ daily, with a maximum daily dose of $15 \mathrm{mg}$; losartan, at a dose of $0.7 \mathrm{mg} / \mathrm{kg}$ up to $1.4 \mathrm{mg} / \mathrm{kg}$ daily-twice daily, with a maximum daily dose of $100 \mathrm{mg}$ ). If HTN could not be brought under control, the treatment was changed to losartan/ hydrochlorothiazide (for hydrochlorothiazide, the dose was 1-2 $\mathrm{mg} / \mathrm{kg} / \mathrm{day}$ in 1 to 2 divided doses, with a maximum daily dose of $50 \mathrm{mg} /$ day) as the second step. Amlodipine was added to the treatment in the third step if HTN could still not be controlled. In patients whose HTN was treated with pharmacotherapy, BP was checked after the first month of therapy. If office BP was still within the hypertensive range, ABPM was performed. The drug dose was increased when all mean BP values were not under the $95^{\text {th }}$ percentile or BP load $>25 \%$. After 1 month of adjusting each drug dose, patients were re-evaluated for their BP. After HTN was brought under control, BP was checked in the second and fourth months during ambulatory visits. Patients who had BP below the 95th percentile after 4 months of treatment (lifestyle changes \pm pharmacotherapy) were evaluated once again with $A B P M$, echocardiography, and biochemical analysis.

At the end of the study, we divided the patients into 2 groups according to their post-treatment (postT) BP: normotensive patients and hypertensive patients. The patients who met normotension criteria were labeled normotensive. The patients who did not meet normotension criteria were identified as hypertensive. Pre- and postT parameters and changes in cardiac and vascular parameters were compared.

\section{ABPM and pulse wave analysis}

Twenty-four-hour ABPM was performed using Mobil-0-Graph pulse wave analysis (PWA)/ABPM CP3120 monitors (IEM, Stolberg, Germany). Monitors were programmed to obtain BP readings every $20 \mathrm{~min}$ from 07:00 to 22:00 and every $30 \mathrm{~min}$ from 22:00 to 07:00. Wake and sleep periods for ABPM were determined from the patients' own statements, and BP was analyzed using the Mobil-0-Graph software (ABP Hypertension Management System CS ver.; IEM). An appropriate cuff was placed on the nondominant arm by a physician, who also informed the child and the parents about how to use the monitoring system.

The device was able to perform measurements such as cBP, pulse pressure amplification, cardiac output (CO), augmentation index corrected for a heart rate of 75 beats per min (Alx@75), PWV, peripheral resistance, and ambulatory arterial stiffness index (AASI). BP measurement of the device was approved by the British Hypertension Society and the European Society of Hypertension and was found to be accurate for PWA measurement in a properly conducted study of invasive catheterization, magnetic resonance imaging, and tonometry devices (5). Reference values published by Elmenhorst et al. (6) were used to derive sex and height-specific z scores for PWV and central systolic BP (cSBP). The number of measurements, means arterial pressure, pulse pressure, systolic and diastolic load, and dipping were evaluated by ABPM, while BP load, dipping status, and AASI values were evaluated by related literature (7-9). 


\section{Echocardiography}

Echocardiographic measurements were performed by a single, well-trained pediatric cardiologist using either the S5-1 or S8-3 probes of the EPIQ-7 echocardiography device (Philips Healthcare, Andover, Massachusetts) in the supine or left lateral decubitus position. In the view of the parasternal long-axis, the measurement was performed and supported with two-dimensional M-mode echocardiography at the end of the diastole and just below the mitral valve fibers. Measurements of left ventricular end-diastolic dimension (LVEDd), interventricular septal thickness (IVSd), and posterior wall thickness (LVPWd) were obtained according to the recommendations of the American Society of Echocardiography (10). Left ventricular mass (LVM) was calculated using the Devereux equation $[0.8 \times\{1.04 \times$ [(LVEDd + LVPWd + IVSd) 3 - LVEDd3] $\}+0.6 \mathrm{~g}]$ by the echocardiography device automatically (11). The LVMI was obtained by dividing LVM by height to the power of 2.7. Left ventricular hypertrophy (LVH) in children has been defined as LVMI > the 95th percentile for the age (12). Relative wall thickness (RWT) was calculated by the formula: $2 \times$ LVPWd/LVEDd (13). LVH was divided into concentric hypertrophy (RWT $>0.42$ ) and eccentric hypertrophy (RWT $\leq 0.42$ ) according to RWT.

\section{cIMT measurements}

A single, well-trained radiologist performed ultrasound scanning using B-mode, color Doppler and pulse wave Doppler sonography of the bilateral carotid arteries with a 4-12 MHz linear array transducer with $4 \mathrm{~cm}$ depth and sound settings optimized to image quality. A frequency of $8 \mathrm{MHz}$ and its harmonic frequencies were used. All examinations were performed using the same protocol (compound imaging, edge enhancement, and standard B-mode greyscale images). The examination was performed with an Aplio 300 ultrasound machine (Toshiba Medical Systems Co, Ltd, Saronno, Italy). After a minimum of 10 min of rest, all patients were examined in the supine position with the neck extended and the head tilted slightly toward the other nonexamined side. cIMT was measured $1-2 \mathrm{~cm}$ below the bifurcation on the right and left common carotid arteries. cIMT was defined as the distance between the lumen-intima interface (upper border) and the media-adventitia interface (lower border) of the far wall and was measured manually using the caliper method. A total of 3 measurements from the 3 segments (distal common carotid, carotid bulb, and proximal internal carotid) were obtained on each side at intervals of approximately 1-2 cm and averaged for each patient. The dynamic range was chosen as $40 \mathrm{~dB}$. The cIMT values were converted into $z$ scores according to the age and height for patients aged 6 years and above (14).

\section{Statistical analysis}

All statistical calculations were done using SPSS for Windows 24.0. All parameters were compared between the preand postT periods using the Wilcoxon test for non-normal distribution variables and paired $t$-test for normal distribution variables. Comparisons of normotensive/hypertensive and normal weight/obese-overweight were made using the Mann-Whitney $U$ test. Discrete variables are expressed as counts (percentages), whereas continuous variables are expressed as medians and quartiles (01-03). Furthermore, a correlation analysis (Pearson correlation coefficient) was also used in the study. Variables with significant correlation with LVMI z scores were then included in the step-wise multiple regression analysis. Statistical significance was defined as $p<0.05$.

\section{Results}

Clinical and demographic characteristics in preT and postT

Thirty-two of 104 patients (17 male; 15 female) diagnosed with primary HTN were enrolled in this study. Isolated systolic HTN was present in $12.5 \%$ of the patients, and systolic and diastolic HTN was present in $87.5 \%$. The demographic and clinical characteristics of the patients are presented in Table 1. Five $(15.6 \%)$ patients presented cIMT more than the $95^{\text {th }}$ percentile; all 5 patients had normal BMI, and 1 had LVH according to ESH2016. Six patients (18\%) were treated by lifestyle changes exclusively, whereas 11 patients (34\%) were prescribed enalapril, 10 patients $(31 \%)$ losartan, 2 patients $(6 \%)$ losartan and hydrochlorothiazide, and 3 patients $(9 \%)$ both losartan-hydrochlorothiazide and amlodipine. In one patient taking losartan and hydrochlorothiazide, an increase in serum creatinine parallel to the increase of dose was found. To address this, amlodipine was included in their treatment, and the dose was not increased. Compared with preT, creatinine, urea, systolic blood pressure (SBP), diastolic BP (DBP), systolic load, diastolic load, cSBP, cSBP z score, central DBP (cDBP), PWV z score, and AASI daytime were regressed. LVM z score, LVMI z score, BMI z score, RWT, ejection fraction (EF), fractioning of shortening (FS), total cholesterol, fasting glucose, uric acid, and Alx@75 were not changed (Table 1). After 6 months, BP was lowered below the $90^{\text {th }}$ percentile in 25 patients $(78.1 \%)$. Systolic BP was between the $90^{\text {th }}-95^{\text {th }}$ percentile in 4 patients and was in stage 1 HTN in 1 patient (15.6\%). The systolic and diastolic BP was between the $90^{\text {th }}-95^{\text {th }}$ percentile in 2 patients $(6.3 \%)$.

\section{Comparison of normal weight and obese/overweight patients}

There was no significant difference in age, sex, cIMT z scores, preT creatinine, changes of EF and FS between normal weight and obese/overweight patients (Table 2). For the obese/ overweight patients, the right cIMT value was $0.42 \mathrm{~mm}(0.34$ $0.47)$ and left cIMT value was $0.4 \mathrm{~mm}(0.36-0.48)$. For the normalweight patients, the right cIMT value was $0.4 \mathrm{~mm}(0.39-0.52)$ and left cIMT value was $0.38 \mathrm{~mm}$ (0.37-0.53). There were no differences in right and left clMT values between the 2 groups ( $p=0.346$ vs. $p=0.623$, respectively). Stage $2 \mathrm{HTN}$ percent, LVMI z score, cSBP z score, PWV z score, and serum uric acid level were higher in obese/overweight patients than in those of normal weight. The percentage of patients with RWT>0.42 in both preT and postT was higher for those with normal weight. LVH in accordance with ESHG16 was regressed only in the normalweight group. All patients in the normal-weight group and $58.8 \%$ 


\begin{tabular}{|c|c|c|c|}
\hline Parameters & Pretreatment & Post-treatment & $P$-value \\
\hline Age (years) & $13.2(11.9-14.9)$ & $14.3(12.7-15.6)$ & $<0.001$ \\
\hline BMI (kg/m²) & $21.6(17.72-25.47)$ & $22.1(17.54-25.34)$ & $0.120^{*}$ \\
\hline BMI z score & $1.31(0.4-1.99)$ & $1.29(0.34-1.92)$ & 0.188 \\
\hline Obesity (\%) & $17(53 \%)$ & $19(59 \%)$ & \\
\hline LVM (g) & $137.2(103.8-157.1)$ & $118.2(107.2-160.5)$ & 0.550 \\
\hline LVM z score & $0.5[(-0.61)$ to $(1.2)]$ & $0.34[(-0.23)-1.27]$ & 0.428 \\
\hline LVMI $\left(\mathrm{g} / \mathrm{m}^{2.7}\right)$ & $36(31-44)$ & $36(33-39.6)$ & 0.763 \\
\hline LVMI z score & $0.99(0.61-1.88)$ & $1.03(0.67-2.32)$ & 0.210 \\
\hline LVMI (g/m²) & $81.92(67.85-91.11)$ & $82.39(69.93-89.39)$ & 0.198 \\
\hline RWT & $0.48(0.36-0.52)$ & $0.44(0.36-0.5)$ & 0.124 \\
\hline LVH (LVMI >38 g/m2.7) & $46.8 \%$ ( $n=15 ;$ obese, $66.6 \%)$ & $25 \%$ (n=8; obese, $75 \%)$ & $0.001^{* * *}$ \\
\hline LVH ESHG16 & $25 \%$ (n=8; obese, $62.5 \%)$ & $18.8 \%$ (n=6; obese, $83.3 \%$ ) & $<0.001^{* *}$ \\
\hline LVH AAPG17 & $3.1 \%(n=1 ;$ obese, $100 \%)$ & & \\
\hline EF (\%) & $69(68-72)$ & $68(66-74)$ & 0.686 \\
\hline FS $(\%)$ & $39(37-40)$ & $40(37-44)$ & 0.993 \\
\hline Creatinine (mg/dL) & $0.57(0.41-0.69)$ & $0.54(0.42-0.61)$ & $<0.001^{*}$ \\
\hline Urea (mg/dL) & $25(18-30)$ & $19(18-21)$ & $<0.001^{*}$ \\
\hline Cholesterol (mg/dL) & $145(131-160)$ & $155(127-160)$ & $0.705^{*}$ \\
\hline Triglycerides (mg/dL) & 104 (78-108) & $108(69-149)$ & $0.630^{*}$ \\
\hline Fasting glucose (mg/dL) & $88(83-94)$ & 90 (78-99) & $0.633^{*}$ \\
\hline Uric acid (mg/dL) & $5.73(4.33-7.67)$ & $5.58(4.2-7.8)$ & $0.128^{*}$ \\
\hline Hemoglobin $(\mathrm{g} / \mathrm{dL})$ & $13.36(11.4-13.32)$ & $13.22(11.5-14.94)$ & 0.084 \\
\hline Microalbuminuria (mg/g creatinine) & $12.8(7.6-18)$ & $11.2(4.4-18)$ & 0.578 \\
\hline NoV measurements & $63(49-65)$ & $59(51-66)$ & 0.061 \\
\hline Systolic BP (mm Hg) & $132(129-137)$ & $113(110-117)$ & $<0.001^{*}$ \\
\hline Diastolic BP (mm Hg) & $81(78-84)$ & $63(59-67)$ & $0.002^{*}$ \\
\hline Systolic load \% & $28(21-45)$ & $21.1(15.8-45)$ & $<0.001^{*}$ \\
\hline Diastolic load \% & 24 (18-29) & 17 (8-25) & $<0.001^{*}$ \\
\hline Systolic non-dipping (<10\%) & $75 \%(n=24)$ & 0 & \\
\hline Diastolic non-dipping (<10\%) & $12.5 \%(n=4)$ & $28.1 \%(n=9)$ & \\
\hline Pulse & 88 (81-93) & 91 (77-93) & 0.972 \\
\hline Pulse pressure & $50(47-56)$ & $49(45-52)$ & 0.078 \\
\hline $\mathrm{cSBP}(\mathrm{mm} \mathrm{Hg})$ & $108(104-110)$ & 102 (97-105) & $0.029 *$ \\
\hline cSBP z score & $1.33(0.28-2.38)$ & $0.93[-0.03)-1.89]$ & $<0.001$ \\
\hline cDBP $(\mathrm{mm} \mathrm{Hg})$ & $73(69-76)$ & $68(62-69)$ & $0.002^{*}$ \\
\hline Alx@75\% & $22.8(18.4-24.9)$ & $22(17.3-24.7)$ & 0.483 \\
\hline $\operatorname{PWV}\left(\mathrm{m} / \mathrm{sn}^{-1}\right)$ & $4.7(4.6-4.8)$ & $4.6(4.5-4.7)$ & 0.071 \\
\hline PWV z score & $0.94(0.1-1.97)$ & $0.8[(-0.04)-1.64]$ & $<0.001$ \\
\hline AASI daytime & $0.69(0.67-0.70)$ & $0.68(0.66-0.70)$ & $<0.001^{*}$ \\
\hline AASI nighttime & $0.70(0.65-0.72)$ & $0.71(0.68-0.77)$ & $0.128^{*}$ \\
\hline \multicolumn{4}{|c|}{ 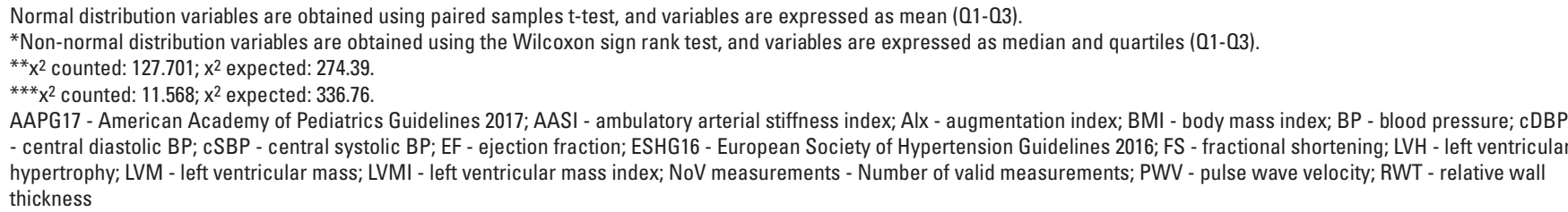 } \\
\hline
\end{tabular}




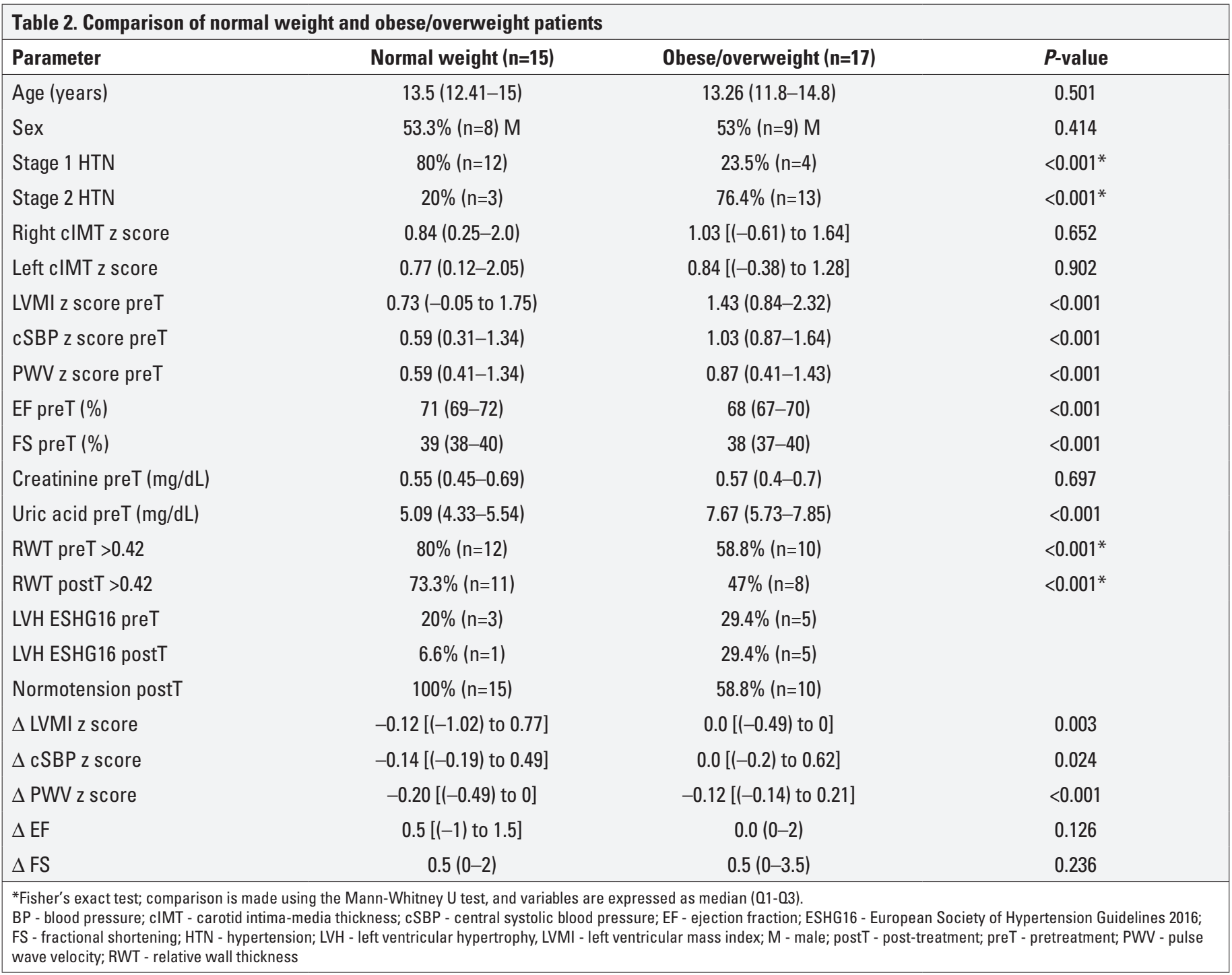

of the obese/overweight group were normotensive at postT. Regression of LVMI z score, cSBP z score, and PWV z score was higher in the normal-weight group than in the obese/overweight group.

\section{Factors associated with LVMI}

While there were significant correlations between SBP, PWV, PWV z score, cIMT z score, and PreT cSBP, there were also correlations between BMI z score, PWV z score, Alx@75, and preT cSBP z score. PreT LVMI z score was correlated with BMI z score, SBP, and PWV z score. Significant correlation analysis results for cSBP, cSBP z score, and LVMI z score are presented in Table 3. A step-wise regression analysis was performed to observe the effect of sex on LVMI z score. We also performed step-wise multiple regression analysis between the 3 covariants with the highest correlation coefficients (Table 4). In the model consisting of male sex, BMI z score, SBP, and PWV $z$ score, $R^{2}$ was found to be 0.336 for LVMI $z$ core $(p<0.001)$.
Comparison of those with and without BP normotension criteria after follow-up

At the end of the study, patients were divided into 2 groups, normotensive and hypertensive. PreT and postT data of the patient groups were then compared. Significant differences based on the comparison of the normotensive and hypertensive patients are presented in Table 5 . At the beginning of the study, hypertensive patients tended to be older and male; their preT BMI z score, LVM z score, LVMI z score, cSBP z score, PWV z score, creatinine, uric acid, and pulse pressure values were higher than those of normotensive patients, and EF and FS were lower. At the end of the study, while the decrease in LVM and LVM z scores of hypertensive patients was smaller than that of normotensive patients, the alteration of EF and FS was not different. While cSBP and PWV z scores decreased in normotensive patients, they increased in hypertensive patients. Of the normotensive patients, six $(24 \%)$ were treated by lifestyle changes only, while 19 patients $(76 \%)$ received 1 antihypertensive medication (enalapril or losartan). Of the hypertensive 


\begin{tabular}{|c|c|c|c|c|c|c|}
\hline \multirow[b]{2}{*}{ Features } & \multicolumn{2}{|c|}{ PreT cSBP } & \multicolumn{2}{|c|}{ PreT cSBP z score } & \multicolumn{2}{|c|}{ PreT LVMI z score } \\
\hline & $\mathbf{r}$ & $P$-value & $\mathbf{r}$ & $P$-value & $\mathbf{r}$ & $P$-value \\
\hline BMI & 0.232 & $<0.001$ & -0.034 & 0.071 & 0.254 & $<0.001$ \\
\hline BMI z score & 0.034 & 0.072 & 0.341 & $<0.001$ & 0.543 & $<0.001$ \\
\hline PWV & 0.780 & $<0.001$ & 0.107 & $<0.001$ & 0.306 & 0.102 \\
\hline PWV z score & 0.509 & $<0.001$ & 0.881 & $<0.001$ & 0.392 & $<0.001$ \\
\hline Alx@75 & -0.165 & $<0.001$ & 0.391 & $<0.001$ & 0.232 & $<0.001$ \\
\hline cIMT z score & 0.338 & $<0.001$ & 0.138 & $<0.001$ & 0.215 & 0.078 \\
\hline
\end{tabular}

Table 4. Step-wise multiple regression results for LVMI z score

\begin{tabular}{lccc|}
\hline Parameters & $\mathbf{R}^{\mathbf{2}}$ & Standardized coefficient B & $\boldsymbol{P}$-value \\
\hline Male sex & 0.037 & 0.268 & $<0.001$ \\
BMI z score & 0.257 & 0.426 & $<0.001$ \\
SBP & 0.305 & 0.069 & 0.005 \\
PWV z score & 0.336 & 0.244 & $<0.001$ \\
\hline
\end{tabular}

Constant: 15.992.

BMI - body mass index; LVMI - left ventricular mass index; PWV - pulse wave velocity; SBP - systolic blood pressure

patients, 2 patients received 1 antihypertensive medication (losartan), 2 patients received 2 antihypertensive drugs (losartan and hydrochlorothiazide), and 3 patients were prescribed a third drug (amlodipine) after 3 months of treatment.

\section{Discussion}

The main finding of our study was that after the BP parameters improved - even while other cardiovascular parameters had not recovered - the cSBP, cSBP z score, and PWV z score values, which are markers of arterial stiffness, regressed.

There are a limited number of studies on the follow-up results for LVH in children treated for primary $\operatorname{HTN}(15,16)$. In 2 of these, LVH that was defined by LVMI above $38.6 \mathrm{~g} / \mathrm{m}^{2.7}$. In a study by Seeman et al. (16), 29 children with primary HTN $(15 \pm 3.7$ years; BMI z score of $1 \pm 1.7 ; 79 \%$ male) were analyzed retrospectively. They found that $52 \%$ of the children had control of HTN according to the BP index. Their study also included patients with secondary HTN. They reported that for all groups, the prevalence of LVH with uncontrolled HTN was higher than with controlled HTN $(46 \%$ vs. $13 \% ; p<0.01)$. Litwin et al. (15) assessed the effect of 12 months of antihypertensive therapy on TOD in 86 children $(14.1 \pm 2.4$ years; $65.1 \%$ overweight; $24.4 \%$ obese; 66 boys). They found BP lowered below the $95^{\text {th }}$ percentile in $74.4 \%$ of the patients. Although our study had a shorter follow-up period and lower normotension levels, we found that $78.1 \%$ of our patients had BP below the $90^{\text {th }}$ percentile. They reported that the proportion of LVH decreased from $46.5 \%$ to $31.4 \%$ after 12 months. In our study, according to a cut-off level of $38 \mathrm{~g} / \mathrm{m}^{2.7}$, the ratio of LVH decreased from $46.8 \%$ to $25 \%$ after 6 months. The differences between the studies in the rates of both $\mathrm{BP}$ and $\mathrm{LVH}$ reduction could have 2 possible explanations. One is that their study population includes more obese and overweight patients $(89.5 \%)$ than ours $(53 \%)$. The other probable explanation is treatment modality. In their study, $57 \%$ of the patients received pharmacological treatment, while in our study, $82 \%$ of the patients were treated with antihypertensives. These results support the effect of more intensive BP control in LVH regression.

In children with HTN, a major pharmacological treatment indication is TOD. According to current guidelines, as a TOD parameter, LVH reversal is a major treatment goal. However, there are different definitions for $\operatorname{LVH}(1,2,12,17-19)$. In addition, LVH is not only affected by BP but also by age, sex, height, race, $\mathrm{BMI}$, fat mass, and lean mass (20-23). In our study, regression analysis revealed that the main predictors of LVMI $z$ score were male sex, BMI z score, SBP, and PWV z score, explaining about $33.6 \%$ in concordance with literature. For this reason, recent guidelines encourage research on subclinical organ damage parameters such as $\mathrm{cBP}$.

Unfortunately, independent of the presence of concomitant cardiovascular disease (CVD), one-third of CV events occur in adults with BP levels in the high-normal range (24). Also, patients aged 20-39 years with stage 1 HTN have a significantly higher risk of CVD compared with those with normal BP (25). It is known that the BP trajectory in childhood and adolescence has the strongest effect on BP in young adulthood. As a result, assessment of organ damage parameters other than LVH may gain importance. A metaanalysis of adult longitudinal studies demonstrated the benefit of CBP in predicting cardiovascular events (26). In another metaanalysis, with an average patient age of 49.9 years, it was con- 


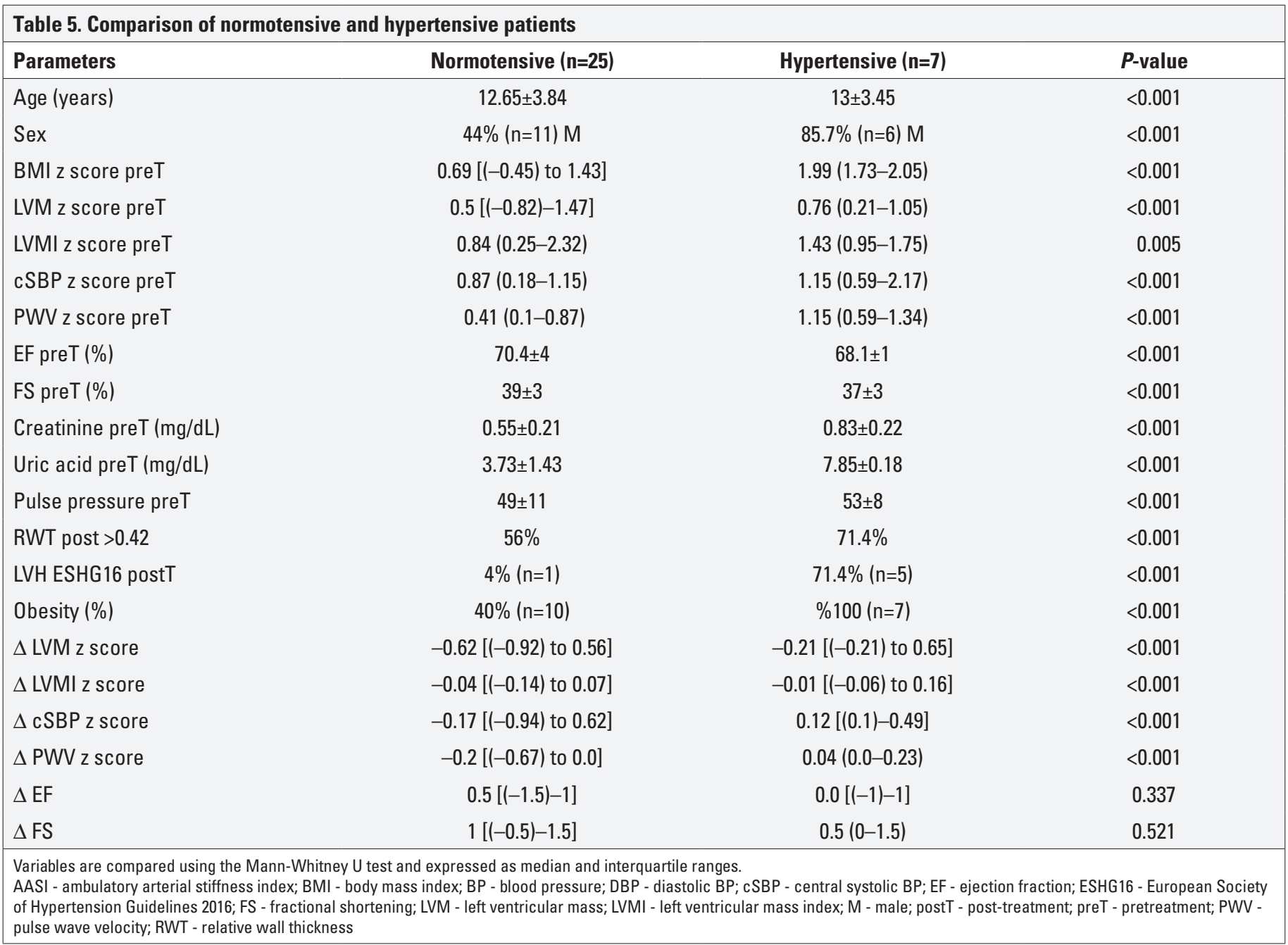

cluded that cBP was more strongly associated with most hypertensive TOD compared to brachial BP (27). Furthermore, it has been shown that cSBP is more closely associated with advanced TOD than brachial BP (28). PWV is accepted as another early subclinical CVD risk factor and is a marker of arterial stiffness in adults (29). Although arterial stiffness has been linked with cardiovascular events in adults, there is little data on such an association in pediatric studies (30). Litwin et al. (31) found that children with severe ambulatory HTN had higher cSBP and PWV and greater prevalence of LVH than white-coat and prehypertensive children. It was found that one-third of children ( $15 \pm 2.4$ years) with primary HTN had normal cSBP and low risk of TOD. Furthermore, it was indicated that PWA, including $\mathrm{CSBP}$, had the same or higher power as ABPM in predicting the presence of hypertensive TOD (32). It was indicated that BP is the only factor independently and strongly associated with PWV in children in all stage of chronic kidney disease stages (30). However, during antihypertensive treatment, the changes in these parameters and their response to BP change in childhood are not clear. PWV was found to be lower in patients with chronic kidney disease with a normal ABPM profile taking antihypertensive medication compared with untreated cases (33). There are no studies determining the change of cSBP in children with primary HTN after treatment. In our study, we observed that after the 6-month follow-up, BMI z score and LVMI z score did not change; however, cSBP and PWV z scores regressed in parallel with the reduction in BP. These suggest that vascular function tests may detect an earlier response of BP regression in the cardiovascular system. It can be speculated that vascular remodeling is more responsive to the reduction in $\mathrm{BP}$ than cardiac remodeling in children. In our study, we observed that while cSBP and PWV z score regression was present in the normotensive group, both arterial stiffness parameters increased in the hypertensive group. We should also consider that all patients whose HTN continued after follow-up were obese, and most were male. It has been shown that carotid-femoral PWV increases in adolescents and children with HTN and metabolic syndrome (34-36). Although there was no change in LVMI z score in our hypertensive patients, the increase in cSBP and PWV continued. This likely supports the conclusion that both obesity and HTN presence have a greater impact on arterial stiffness than does cardiac remodeling. In addition, when we looked at the basic characteristics of hypertensive patients, they were older and had higher BMI and LVMI z scores. Furthermore, at the beginning of the study, the patients in the obese/overweight group had worse 
cardiovascular and metabolic factors than those of the normalweight group. They probably had stiffer arteries due to both age and BMI at the beginning of the treatment. Litwin et al. (31) found that children (mean age, 15 years) with HTN who had elevated cSBP also had higher cIMT, LVMI, and PWV values. Therefore, the recovery of stiffer arteries may require longer and more intense treatment. The other possible explanation for the lack of increase in LVMI z score is that treatment with RAAS blockade may pause the progression of cardiac remodeling, even though vascular remodeling continues. Besides, before the treatment, normotensive patients had lower BMI scores, were younger, and most were female. Therefore, since the arterial stiffness of patients with a lower CVD risk factor is expected to be less, CSBP and PWV regression may have been achieved earlier with treatment. Finally, in a short-term follow-up in children with low CVD risk, as BP normalizes, regression of cSBP and PWV may occur without LVMI reversal.

HTN is the only correctable CVD risk factor among the many factors that affect LVMI. ABPM is considered more reliable for diagnosing HTN. It was argued that cSBP has greater specificity and sensitivity than ABPM in predicting LVH (31). cSBP and PWV appear more sensitive to BP change and may contribute to LVMI in guiding our treatment. In the HARVEST study, it was indicated that young-to-middle-age patients with isolated systolic HTN with low CBP needed less aggressive HTN treatment (37). In a prospective adult HTN study, it was found that during antihypertensive treatment, cSBP added significant value to clinic SBP for predicting the regression of TOD (33).

Possible reasons for early regression of CSBP and PWV maybe that medium-sized arteries are more elastic in children than in adults, and therefore may result in a decrease in backward pulse wave accumulation when brachial arterial pressure decreases. Although we could not evaluate cIMT after treatment, patients with a cIMT value $>95 p$ before treatment were normotensive after treatment. Another possible reason for the regression of cSBP and PWV is that while atherosclerosis starts in childhood, it may not yet have entered the irreversible vascular remodeling (intimal fibrosis) process, which is difficult to reverse.

\section{Study limitations}

There were some limitations to this study. Follow-up time was short, and the number of patients studied was small. In addition, cIMT could not be evaluated after treatment. We could not use an objective method to evaluate the compliance to antihypertensive treatment but instead relied on verbal statements from patients and families. There is no clear information about the effect of sex on arterial stiffness. However, this is the first longitudinal study of children to investigate whether CSBP and PWV normalize in parallel with BP reduction.

\section{Conclusion}

After BP improved, though LVMI had not yet regressed, the cSBP, cSBP z score, and PWV z score values, which are markers of arterial stiffness, regressed. These parameters may be the first cardiovascular parameters to respond to normotension in childhood. Further longitudinal studies are needed for the assessment of BP control on arterial stiffness in childhood.

Conflict of interest: None declared.

Peer-review: Externally peer-reviewed.

Author contributions: Concept - D.Ö.H., Ö.C., A.H.Y.; Design - D.Ö.H., Ö.C., A.H.Y.; Supervision - D.Ö.H.; Fundings - D.Ö.H.; Materials - D.Ö.H., Ö.C., A.H.Y.; Data collection \&/or processing - D.Ö.H., Ö.C., A.H.Y.; Analysis \&/or interpretation - D.Ö.H.; Literature search - D.Ö.H., Ö.C., A.H.Y.; Writing - D.Ö.H., Ö.C., A.H.Y.; Critical review - D.Ö.H., Ö.C., A.H.Y.

\section{References}

1. Lurbe E, Agabiti-Rosei E, Cruickshank JK, Dominiczak A, Erdine S, Hirth A, et al. 2016 European Society of Hypertension guidelines for the management of high blood pressure in children and adolescents. J Hypertens 2016; 34: 1887-920. [Crossref]

2. Flynn JT, Kaelber DC, Baker-Smith CM, Blowey D, Carroll AE, Daniels SR, et al.; SUBCOMMITTEE ON SCREENING AND MANAGEMENT OF HIGH BLOOD PRESSURE IN CHILDREN. Clinical Practice Guideline for Screening and Management of High Blood Pressure in Children and Adolescents. Pediatrics 2017; 140: e20171904. [Crossref]

3. McEniery CM, Cockcroft JR, Roman MJ, Franklin SS, Wilkinson IB. Central blood pressure: current evidence and clinical importance. Eur Heart J 2014; 35: 1719-25. [Crossref]

4. Urbina E, Alpert B, Flynn J, Hayman L, Harshfield GA, Jacobson M, et al.; American Heart Association Atherosclerosis, Hypertension, and Obesity in Youth Committee. Ambulatory blood pressure monitoring in children and adolescents: recommendations for standard assessment: a scientific statement from the American Heart Association Atherosclerosis, Hypertension, and Obesity in Youth Committee of the council on cardiovascular disease in the young and the council for high blood pressure research. Hypertension 2008; 52: 433-51. [Crossref]

5. Omboni S, Posokhov IN, Kotovskaya YV, Protogerou AD, Blacher J. Twenty-Four-Hour Ambulatory Pulse Wave Analysis in Hypertension Management: Current Evidence and Perspectives. Curr Hypertens Rep 2016; 18: 72. [Crossref]

6. Elmenhorst J, Hulpke-Wette M, Barta C, Dalla Pozza R, Springer S, Oberhoffer R. Percentiles for central blood pressure and pulse wave velocity in children and adolescents recorded with an oscillometric device. Atherosclerosis 2015; 238: 9-16. [Crossref]

7. White WB, Dey HM, Schulman P. Assessment of the daily blood pressure load as a determinant of cardiac function in patients with mild-to-moderate hypertension. Am Heart J 1989; 118: 782-95. [Crossref]

8. White WB, Larocca GM. Improving the utility of the nocturnal hypertension definition by using absolute sleep blood pressure rather than the "dipping" proportion. Am J Cardiol 2003; 92: 1439-41. [Crossref]

9. Dolan E, Li Y, Thijs L, McCormack P, Staessen JA, O'Brien E, et al. Ambulatory arterial stiffness index: rationale and methodology. Blood Press Monit 2006; 11: 103-5. [Crossref]

10. Lang RM, Badano LP, Mor-Avi V, Afilalo J, Armstrong A, Ernande L, et al. Recommendations for cardiac chamber quantification by 
echocardiography in adults: an update from the American Society of Echocardiography and the European Association of Cardiovascular Imaging. J Am Soc Echocardiogr 2015; 28: 1-39. [Crossref]

11. Devereux RB, Alonso DR, Lutas EM, Gottlieb GJ, Campo E, Sachs I, et al. Echocardiographic assessment of left ventricular hypertrophy: comparison to necropsy findings. Am J Cardiol 1986; 57: 450-8. [Crossref]

12. Khoury PR, Mitsnefes M, Daniels SR, Kimball TR. Age-specific reference intervals for indexed left ventricular mass in children. $J$ Am Soc Echocardiogr 2009; 22: 709-14. [Crossref]

13. Ganau A, Devereux RB, Roman MJ, de Simone G, Pickering TG, Saba PS, et al. Patterns of left ventricular hypertrophy and geometric remodeling in essential hypertension. J Am Coll Cardiol 1992; 19: 1550-8. [Crossref]

14. Doyon A, Kracht D, Bayazit AK, Deveci M, Duzova A, Krmar RT, et al.; 4C Study Consortium. Carotid artery intima-media thickness and distensibility in children and adolescents: reference values and role of body dimensions. Hypertension 2013; 62: 550-6. [Crossref]

15. Litwin M, Niemirska A, Sladowska-Kozlowska J, Wierzbicka A, Janas R, Wawer ZT, et al. Regression of target organ damage in children and adolescents with primary hypertension. Pediatr Nephrol 2010; 25: 2489-99. [Crossref]

16. Seeman T, Dostálek L, Gilík J. Control of hypertension in treated children and its association with target organ damage. Am J Hypertens 2012; 25: 389-95. [Crossref]

17. Foster BJ, Khoury PR, Kimball TR, Mackie AS, Mitsnefes M. New Reference Centiles for Left Ventricular Mass Relative to Lean Body Mass in Children. J Am Soc Echocardiogr 2016; 29: 441-7. [Crossref]

18. de Simone G, Devereux RB, Daniels SR, Koren MJ, Meyer RA, Laragh $\mathrm{JH}$. Effect of growth on variability of left ventricular mass: assessment of allometric signals in adults and children and their capacity to predict cardiovascular risk. J Am Coll Cardiol 1995; 25: 1056-62. [Crossref]

19. Chinali M, Emma F, Esposito C, Rinelli G, Franceschini A, Doyon A, et al. Left Ventricular Mass Indexing in Infants, Children, and Adolescents: A Simplified Approach for the Identification of Left Ventricular Hypertrophy in Clinical Practice. J Pediatr 2016; 170: 193-8. [Crossref]

20. Urbina EM, Mendizábal B, Becker RC, Daniels SR, Falkner BE, Hamdani G, et al. Association of Blood Pressure Level With Left Ventricular Mass in Adolescents. Hypertension 2019; 74: 590-6. [Crossref]

21. Juhász $M$, Katona $E$, Settakis G, Paragh G, Molnár C, Fülesdi B, et al. Gender-related differences in adolescent hypertension and in target organ effects. J Womens Health (Larchmt) 2010; 19: 759-65. [Crossref]

22. Daniels SR, Kimball TR, Morrison JA, Khoury P, Witt S, Meyer RA. Effect of lean body mass, fat mass, blood pressure, and sexual maturation on left ventricular mass in children and adolescents. Statistical, biological, and clinical significance. Circulation 1995; 92: 3249-54. [Crossref]

23. Urbina EM, Gidding SS, Bao W, Pickoff AS, Berdusis K, Berenson GS. Effect of body size, ponderosity, and blood pressure on left ventricular growth in children and young adults in the Bogalusa Heart Study. Circulation 1995; 91: 2400-6. [Crossref]
24. Huang Y, Wang S, Cai X, Mai W, Hu Y, Tang H, et al. Prehypertension and incidence of cardiovascular disease: a meta-analysis. BMC Med 2013; 11: 177. [Crossref]

25. Son JS, Choi S, Kim K, Kim SM, Choi D, Lee G, et al. Association of Blood Pressure Classification in Korean Young Adults According to the 2017 American College of Cardiology/American Heart Association Guidelines With Subsequent Cardiovascular Disease Events. JAMA 2018; 320: 1783-92. [Crossref]

26. Vlachopoulos C, Aznaouridis K, O'Rourke MF, Safar ME, Baou K, Stefanadis C. Prediction of cardiovascular events and all-cause mortality with central haemodynamics: a systematic review and meta-analysis. Eur Heart J 2010; 31: 1865-71. [Crossref]

27. Kollias A, Lagou S, Zeniodi ME, Boubouchairopoulou N, Stergiou GS. Association of Central Versus Brachial Blood Pressure With Target-Organ Damage: Systematic Review and Meta-Analysis. Hypertension 2016; 67: 183-90. [Crossref]

28. McEniery CM, Cockcroft JR, Roman MJ, Franklin SS, Wilkinson IB. Central blood pressure: current evidence and clinical importance. Eur Heart J 2014; 35: 1719-25. [Crossref]

29. Naka KK, Papathanassiou K, Bechlioulis A, Pappas K, Tigas S, Makriyiannis $D$, et al. Association of vascular indices with novel circulating biomarkers as prognostic factors for cardiovascular complications in patients with type 2 diabetes mellitus. Clin Biochem 2018; 53: 31-7. [Crossref]

30. Schaefer F, Doyon A, Azukaitis K, Bayazit A, Canpolat N, Duzova A, et al.; 4C Study Consortium. Cardiovascular Phenotypes in Children with CKD: The 4C Study. Clin J Am Soc Nephrol 2017; 12: 19-28. [Crossref]

31. Litwin M, Obrycki $Ł$, Niemirska A, Sarnecki J, Kułaga Z. Central systolic blood pressure and central pulse pressure predict left ventricular hypertrophy in hypertensive children. Pediatr Nephrol 2019; 34: 703-12. [Crossref]

32. Shimizu M, Hoshide S, Ishikawa J, Yano Y, Eguchi K, Kario K. Correlation of Central Blood Pressure to Hypertensive Target Organ Damages During Antihypertensive Treatment: The J-TOP Study. Am J Hypertens 2015; 28: 980-6. [Crossref]

33. Düzova A, Karabay Bayazit A, Canpolat N, Niemirska A, Kaplan Bulut l, Azukaitis K, et al.; 4C Study Consortium. Isolated nocturnal and isolated daytime hypertension associate with altered cardiovascular morphology and function in children with chronic kidney disease: findings from the Cardiovascular Comorbidity in Children with Chronic Kidney Disease study. J Hypertens 2019; 37: 2247-55. [Crossref]

34. Urbina EM, Khoury PR, McCoy C, Daniels SR, Kimball TR, Dolan LM. Cardiac and vascular consequences of pre-hypertension in youth. J Clin Hypertens (Greenwich) 2011; 13: 332-42. [Crossref]

35. Kulsum-Mecci N, Goss C, Kozel BA, Garbutt JM, Schechtman KB, Dharnidharka VR. Effects of Obesity and Hypertension on Pulse Wave Velocity in Children. J Clin Hypertens (Greenwich) 2017; 19: 221-6. [Crossref]

36. Pandit DS, Khadilkar AV, Chiplonkar SA, Khadilkar VV, Kinare AS. Arterial stiffness in obese children: Role of adiposity and physical activity. Indian J Endocrinol Metab 2014; 18: 70-6. [Crossref]

37. Saladini F, Santonastaso M, Mos L, Benetti E, Zanatta N, Maraglino G, et al.; HARVEST Study Group. Isolated systolic hypertension of young-to-middle-age individuals implies a relatively low risk of developing hypertension needing treatment when central blood pressure is low. J Hypertens 2011; 29: 1311-9. [Crossref] 